\section{MS6-P2 Crystal structure of Plasmodium} falciparum calmodulin / peptide complex

Veronika Harmat $^{1,2}$, Zsolt Dürvanger ${ }^{1}$, Tünde Juhász ${ }^{3}$, Károly Liliom $^{3}$

1. Institute of Chemistry, Eötvös Loránd University, Budapest, Hungary

2. MTA-ELTE Protein Modelling Research Group, Budapest, Hungary

3. Institute of Enzymology, Research Centre for Natural Sciences, Hungarian Academy of Sciences, Budapest, Hungary

\section{email: veronika@chem.elte.hu}

Calmodulin, a ubiquitous calcium binding protein is present in all eukaryotic cells. It regulates the action of more than 100 proteins by interacting with them in a calcium dependent way. Both of its lobes contain two calcium binding EF-hand motifs. $\mathrm{Ca}^{2+}$ binding of the protein causes a global conformational change and exposure of hydrophobic patches on both domains capable for binding target proteins. Though the bound segments of the target possess basic amphiphilic helix conformation, the target orientation, distances of their anchoring residues and orientations of the calmodulin lobes varies within the complexes. Some calmodulin antagonist compounds were reported to inhibit Plasmodium falciparum, suggesting $P$. falciparum calmodulin could be a possible target of anti-malarial treatment. Structures of calmodulin and apocalmodulin from several species are known, but that of $P$. falciparum has not been published. Though calmodulin is a highly conserved protein, its structure explores differences between vertebrate and $P$. falciparum calmodulin.

We solved the structure of calmodulin from $P$. falciparum in complex with a model target peptide (melittin, a component of bee venom) and refined the structure to $2.4 \mathrm{~A}$ resolution. The structure contains four calmodulin/melittin complex units showing two main binding modes of the peptide: there are some differences in the orientations of calmodulin lobes and binding patterns for the peptide. It is the flexibility of the middle short coil segment within the melittin helix that facilitates different binding modes to calmodulin. The structure suggests that interactions with the two calmodulin lobes are formed independently.

This study was supported by the MedInProt program of the Hungarian Academy of Sciences; OTKA grants NK101072 to V.H, PD104344 to T.J. and K82092 to K.L. We acknowledge Paul Scherrer Institut, Villigen, Switzerland for providing synchrotron radiation beamtime, and Gergó Gógl and Péter Ecsédi for collecting data. The research leading to these results has received funding from the European Community's $7^{\text {th }}$ Framework Programme (FP7/2007-2013) under BioStruct-X (grant agreement No.283570).

Keywords: calcium signalling, protein/peptide complex, flexible binding, Plasmodium falciparum

\section{MS6-P3 The molecular bases of $\delta \beta$ T-cell mediated antigen recognition}

Stephanie Gras ${ }^{1,2}$, Eric Chabrol $^{1}$, Sidonia B.G. Eckle ${ }^{3}$, Renate de Boer $^{4}$, James McCluskey ${ }^{3}$, Jamie Rossjohn ${ }^{1,2,5}$, Mirjam H.M. Heemskerk $^{4}$

1. Department of Biochemistry and Molecular Biology, School of Biomedical Sciences, Monash University, Clayton, Victoria 3800, Australia

2. Australian Research Council Centre of Excellence for Advanced Molecular Imaging, Monash University, Clayton, Victoria 3800, Australia

3. Department of Microbiology and Immunology, Peter Doherty Institute for Infection and Immunity, University of Melbourne, Parkville, Victoria 3010, Australia

4. Leiden University Medical Center, Department of Hematology, C2-R, P.O. Box 9600, 2300 RC Leiden, The Netherlands

5. Institute of Infection and Immunity, Cardiff University, School of Medicine, Heath Park, Cardiff CF14 4XN, UK

email: stephanie.gras@monash.edu

$\alpha \beta$ and $\gamma \boldsymbol{\delta}$ T-cells are disparate T-cell lineages that, via their use of either $\alpha \beta$ or $\gamma \delta$ T-cell antigen receptors (TCRs) respectively, can respond to distinct antigens. Here we characterise a new population of human T-cells, term $\boldsymbol{\delta} \boldsymbol{\beta}$ T-cells, that express TCRs comprising a TCR- $\boldsymbol{\delta}$ variable gene fused to a Joining- $\alpha /$ Constant- $\alpha$ domain, paired with an array of TCR- $\boldsymbol{\beta}$ chains. We characterised the cellular, functional, biophysical and structural characteristic feature of this new T-cells population that reveal some new insight into TCR diversity. We provide molecular bases of how $\boldsymbol{\delta} \boldsymbol{\beta}$ T-cells can recognise viral peptide presented by Human Leukocyte Antigen (HLA) molecule. Our findings highlight how components from $\alpha \beta$ and $\gamma \delta$ TCR gene loci can recombine to confer antigen specificity thus expanding our understanding of T-cell biology and TCR diversity.

Keywords: immunology, new T cell receptor, HLA 